Research Paper

\title{
The Effect of Different Concentrations of Methylprednisolone on Survival, Proliferation, and Migration of Neural Stem/Progenitor Cells
}

\author{
Zohreh Bagheri' ${ }^{1}$, Fatemeh Shamsi ${ }^{2,3}$, Zahra Zeraatpisheh $^{2,3}$, Mahin Salmannejad ${ }^{4}$, Ahmad Soltani $^{5}$, Hadi Aligholi $^{2,3,6^{*}}$ (1) \\ 1. Department of Physiology, School of Medicine, Shiraz University of Medical Sciences, Shiraz, Iran. \\ 2. Department of Neurosciences, School of Advanced Medical Sciences and Technologies, Shiraz University of Medical Sciences, Shiraz, Iran. \\ 3. Neuroscience Laboratory (Brain, Cognition and Behavior), Department of Neurosciences, School of Advanced Medical Sciences and Technologies, \\ Shiraz University of Medical Sciences, Shiraz, Iran. \\ 4. Department of Anatomy, School of Medicine, Shiraz University of Medical Sciences, Shiraz, Iran. \\ 5. Department of Neurosurgery, Namazi Hospital, Shiraz University of Medical Sciences, Shiraz, Iran \\ 6. Epilepsy Research Center, Shiraz University of Medical Sciences, Shiraz, Iran.
}

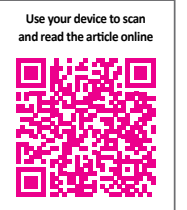

cittation Bagheri, Z., Shamsi, F., Zeraatpisheh, Z., Salmannejad, M., Soltani, A., Aligholi, H. (2022). The Effect of Different Concentrations of Methylprednisolone on Survival, Proliferation, and Migration of Neural Stem/Progenitor Cells. Basic and Clinical Neuroscience, 13(2), 207-214. http://dx.doi.org/10.32598/bcn.2021.35.4

\section{(c) (1) (\$)}

Article info:

Received: 17 Jan 2020

First Revision: 13 Apr 2020

Accepted: 26 Jul 2020

Available Online: 01 Mar 2022

Keywords:

Methylprednisolone, Neural stem cells, Proliferation,

Survival, Migration

\section{A B S T RA C T}

Introduction: The present study addressed whether methylprednisolone (MP) as an antiinflammatory drug used in neurodegenerative diseases and neural stem/progenitor cells (NS/ $\mathrm{PCs}$ ) is safe.

Methods: First, embryonic rat NS/PCs were exposed to different concentrations of MP, and then we evaluated their survival by MTT assay, proliferation by analyzing the number and diameter of neurospheres, and the migration of the cells by neurosphere assay.

Results: The viability of NS/PCs was reduced following exposure to 10,15 , and $20 \mu \mathrm{g}$ / $\mathrm{mL}$ of MP. In addition, although the number of neurospheres did not change, exposure to different concentrations of MP resulted in the formation of smaller neurospheres. Despite these undesirable effects, the highest concentration of MP $(20 \mu \mathrm{g} / \mathrm{mL})$ increased the migration capacity of the NS/PCs.

Conclusion: The combination of MP and NS/PCs is not recommended due to the adverse effects of MP on the survival and proliferation of NS/PCs.

\section{* Corresponding Author:}

Hadi Aligholi, PhD.

Address: Department of Neurosciences, School of Advanced Medical Sciences and Technologies, Shiraz University of Medical Sciences, Shiraz, Iran. Tel: +98 (71) 32305471

E-mail:aligholi@sums.ac.ir 


\section{Highlights}

- Methylprednisolone reduced survival of neural stem/progenitor cells.

- Methylprednisolone decreased proliferation of neural stem/progenitor cells.

- The highest concentration of MP $(20 \mu \mathrm{g} / \mathrm{mL})$ increased the migration capacity of the neural stem/progenitor cells.

\section{Plain Language Summary}

In this study, we evaluate the effect of the exposure of neural stem/progenitor cells to methylprednisolone. Based on the results, combination of neural stem/progenitor cells and methylprednisolone not recommended due to reduction of survival and proliferation of the cells.

\section{Introduction}

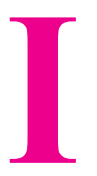

nflammation is one of the major events that contribute to the pathology of neurodegenerative diseases such as spinal cord injury (SCI), Alzheimer disease, Parkinson disease, multiple sclerosis, and epilepsy (Gao \& Hong, 2008). In other words, immune system activation seen in neurodegenerative disease has photogenic and supportive roles (Amor, Puentes, Baker, \& Van Der Valk, 2010). Hence, inflammation management is an essential issue in treating neurodegenerative diseases.

One of the primary drugs administered to manage inflammation following neurodegenerative disorders is methylprednisolone (MP). For instance, an intravenous injection of a high concentration of MP $(30 \mathrm{mg} / \mathrm{kg}$ of body weight in the first time followed by $4.5 \mathrm{mg} / \mathrm{kg} / \mathrm{h}$ for 23 hours) is used during the first hours after SCI to reduce the secondary injury (Bracken et al., 1990). In addition, this drug has been reported as a promising approach for treating Alzheimer disease (Alisky, 2008), Parkinson disease (Sato, Asoh, Metoki, \& Satoh, 2003), multiple sclerosis (Saidha, Mok, Butler, Fanning, \& Harrington, 2010), stroke (Altamentova et al., 2020), and epilepsy (Almaabdi et al., 2014). Although some neurological improvements have been reported following the administration of MP, the systemic administration of a high dose of the drug shows important side effects, including wound infection, pneumonia, gastrointestinal bleeding, and myopathy (Gerndt et al., 1997; Qian et al., 2005). Thus, changing the route of administration from systemic to local might be a promising approach for decreasing these adverse effects of MP.
On the other hand, the treatment of neurodegenerative diseases goes beyond pharmacotherapy, and new approaches like applying neural stem/progenitor cells (NS/ PCs) are in progress (Ronaghi, Erceg, Moreno-Manzano, \& Stojkovic, 2010; Russo, 2020). Several basic investigations indicate that transplanted NS/PCs survive, migrate, and differentiate into neurons, astrocytes, and oligodendrocytes (Aligholi et al., 2016; Cummings et al., 2005). In addition, NS/PCs release trophic factors such as nerve growth factors and brain-derived neurotrophic factors, which can be helpful for neuroregeneration ( $\mathrm{Lu}$, Jones, Snyder, \& Tuszynski, 2003). Moreover, functional recovery has been reported after transplantation of $\mathrm{NS} / \mathrm{PCs}$ to the damaged tissue of the spinal cord (Sankavaram et al., 2019). Despite these outstanding properties of NS/PCs, their transplantation must be combined with other agents to modulate their behavior (Garbossa, Boido, Fontanella, Fronda, Ducati, \& Vercelli, 2019).

Based on the above statements, the combination of NS/ PCs and MP has been considered. In this sense, what is needed to be clear is the effect of MP on the behavior of NS/PCs. One study reported that administration of MP after brain ischemia increased survival and migration of NS/PCs (hong Jing, Ping Hou, Feng Song, \& Yin, 2012). Using MP in a brain injury model showed that this drug supported oligodendrocytes but did not affect the survival of neurons (Lee et al., 2008). In addition, the proliferation of NS/PCs in an SCI model decreased by MP (Obermair, Schroter, \& Thallmair, 2008). In an in vitro study, a reduction in proliferation of spinal cord-derived NS/PCs after exposure to MP was reported (Wang et al., 2014). Due to these discrepancies, the present study evaluated the effects of different concentrations of MP on survival, proliferation, and migration of rat embryonic NS/PCs using neurosphere assay. 


\section{Materials and Methods}

All methods were performed in accordance with the institutional guidelines of Shiraz University of Medical Sciences for animal care and use.

\section{Culture of NS/PCs}

The ganglionic eminence of a 13.5-day-old rat embryo was harvested using a stereomicroscope. After mechanical cutting by surgical knife, the specimens were dissociated using $0.05 \%$ trypsin/EDTA (Invitrogen, USA) for 5 $\min$ at $37^{\circ} \mathrm{C}$. Then, a soybean trypsin inhibitor was added (Sigma, USA). After centrifugation and discarding the supernatant, the single cells were plated in Dulbecco's modified Eagle's medium/F12 (Invitrogen, USA) containing $1 \%$ N2 supplement (Invitrogen, USA), 2\% B27 supplement (Invitrogen, USA), 1\% penicillin/streptomycin, 1\% glutaMax (Invitrogen, USA), $20 \mathrm{ng} / \mathrm{mL}$ epidermal growth factor (EGF; Miltenybiotech, Germany), and incubated at $37^{\circ} \mathrm{C}$ and $5 \% \mathrm{CO} 2$. During the following days, NS/ PCs proliferated as free-floating clusters (neurospheres). When the diameter of the spheres became about $200 \mu \mathrm{m}$, subculturing was done, and the cells were replated into a fresh growth medium. Following the second passage, the obtained NS/PCs were used for the study.

\section{Study design}

The cells obtained from the second passage $(3 \times 104$ cells per well) were cultured in 96-well plates and used for investigation. A range of $0.25 \mu \mathrm{g} / \mathrm{mL}$ to $1000 \mu \mathrm{g} / \mathrm{mL}$ of MP was used in MP-based previous in vitro studies (Kuppermann, Zacharias, \& Kenney, 2014; Mealey, Chen, \& Schanz, 1971; Wang et al., 2014). In the present investigation, we selected concentrations of $0,5,10,15$, or $20 \mu \mathrm{g} / \mathrm{mL}$ of MP (Sigma, USA) due to the toxicity of this drug in higher concentrations for NS/PCs (according to our pilot study). Cell viability, proliferation, and migration of NS/PCs were evaluated using the following methods (4 well/group).

\section{Cell viability assessment}

The NS/PCs were exposed to different concentrations of MP for 7 days. Then, an MTT assay was done to evaluate cell viability. Briefly, the cells were incubated with MTT solution for 4 hours; then, the reaction was ceased by dimethyl sulfoxide (DMSO). Absorbance was measured by an ELISA microplate reader at $570 \mathrm{~nm}$.

\section{Proliferation assay}

Two parameters of the number and the diameter of neurospheres were considered proliferation index. The NS/PCs were cultured in 96-well plates containing the neurosphere medium (3 wells per group). The number of neurospheres in each well was calculated on days 3 , 5 , and 7 under an inverted microscope (Optika, Italy). Moreover, the diameter of neurospheres was measured in 5 photos taken from the corners and center of each well, using Infinity software on days 3,5 , and 7 . The average diameter of two diagonals perpendicular to each other was reported as the neurosphere diameter.

\section{Cell migration assay}

To evaluate the migration of the cells, the neurospheres were cultured in poly-L-ornithine-treated plates, and then the migration was monitored by taking photos on days $1,3,5$, and 7 . The average distance passed by the three cells located at the farthest distance to the margin of the neurosphere was measured by Infinity software as an index of cell migration.

\section{Statistical analysis}

The study data are presented as Mean \pm SD. The normal distribution of data was tested prior to statistical analysis. One-way analysis of variance (ANOVA) was used if data were distributed normally and the Kruskal-Wallis ANOVA test (KWT) for nonparametric data. The least significant difference was used as a post hoc test. $\mathrm{P}<0.05$ was considered statistically significant.

\section{Results}

\section{Primary culture}

The isolated ganglionic eminence was cultured in a serum-free medium. As indicated in Figure 1, the NS/ PCs were proliferated as free-floating neurospheres. One week after primary culturing, the diameter of the neurospheres was more than $100 \mu \mathrm{m}$; thus, passaging was done. The single cells obtained from the second passage were used for the rest of the study.

\section{The effect of MP on the viability of the NS/PCs}

The results of the MTT assay on day 7 post-treatment showed that following exposure to 10,15 , and $20 \mu \mathrm{g} /$ $\mathrm{mL}$ of MP, the survival of the NS/PCs decreased significantly compared to that of the group without any drug exposure $(\mathrm{P}<0.05)$. In contrast, the cell viability did not change by $5 \mu \mathrm{g} / \mathrm{mL}$ of MP (Figure 2 ). 

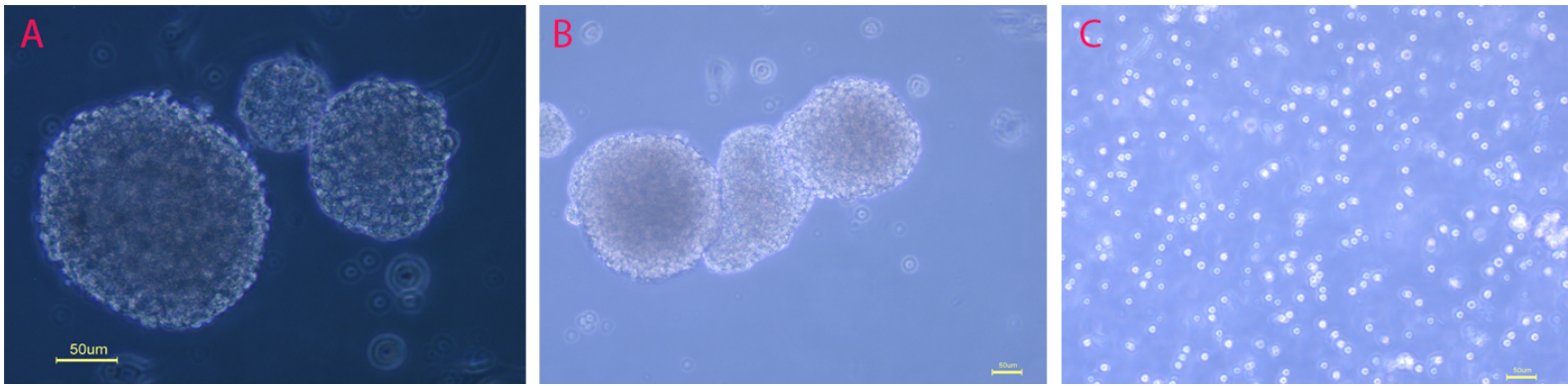

Figure 1. Culture of neural Stem/Progenitor cells (NS/PCs)

NEUR:SCIENCE

The ganglionic eminence was isolated and cultured in a serum-free condition, and after 7 days, the proliferated cells appeared as well-shaped and visible neurospheres (A). After the second passage, the neurospheres (B) were dissociated as single cells (C) and were used for the rest of the study.

\section{The effect of MP on the proliferation of the NS/PCs}

The number of neurospheres produced from the single cells after 3 days was not significantly different between the MP-treated groups and the non-treated group. Although, the number of neurospheres significantly decreased in the $15 \mu \mathrm{g} / \mathrm{mL}$ MP group compared to that of the 5 and $10 \mu \mathrm{g} / \mathrm{mL}$ of MP groups ( $\mathrm{P}<0.05$, Figure $3 \mathrm{~F}$ ). On days 5 and 7 of post-treatment of the NS/PCs with MP, there was no significant difference in the number of neurospheres between the groups. On the other hand, the diameter of neurospheres dramatically reduced following exposure of the NS/PCs to different concentrations of MT compared to the non-treated group. This effect could be observed 3, 5, and 7 days after exposure to MP $(\mathrm{P}<0.05)$ except for the concentration of $20 \mu \mathrm{g} / \mathrm{mL}$ of MP on day 5 (Figure $3 \mathrm{G}$ ).

\section{The effect of MP on the migration of the NS/PCs}

As illustrated in Figure 4, an increasing trend could be seen in the migration capacity of the NS/PCs in all groups from day 1 to day 7 following exposure to different concentrations of MP. On days 1 and 3 after exposure, there were no significant differences between the groups in the migration index. Although, the migration of NS/PCs treated with $20 \mu \mathrm{g} / \mathrm{mL}$ of MP was higher than that of the non-treated group on days 5 and 7 following exposure.

\section{Discussion}

In the present study, we indicated that high concentrations of MP threatened the survival of NS/PCs, and the proliferation capacity of NS/PCs decreased by exposure to different concentrations of MP. However, the highest concentrations of MP $(20 \mu \mathrm{g} / \mathrm{mL})$ enhanced the migration capacity of NS/PCs.

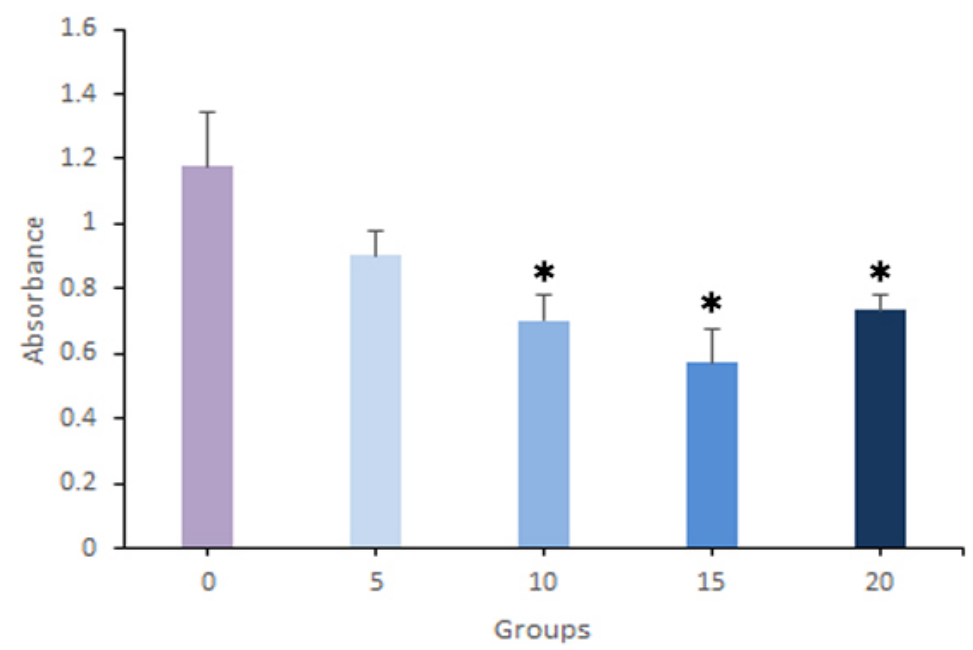

Figure 2. Cell viability assay

The survival of the neural stem/progenitor cells (NS/PCs) was evaluated seven days after exposure to $5,10,15,0$ or $20 \mu \mathrm{g} / \mathrm{mL}$ of methylprednisolone (MP) by MTT assay.*: $\mathrm{P}<0.05$ vs the untreated group. 

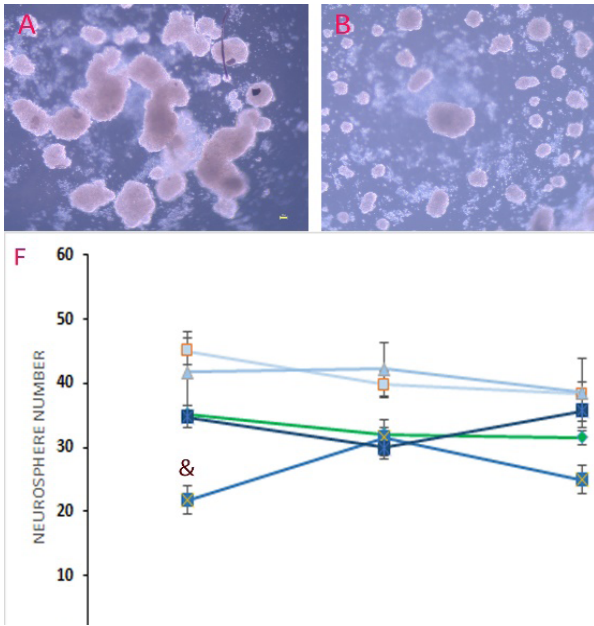

Figure 3. Cell proliferation assay
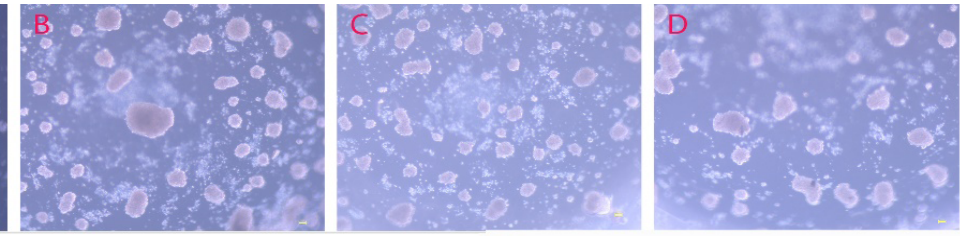

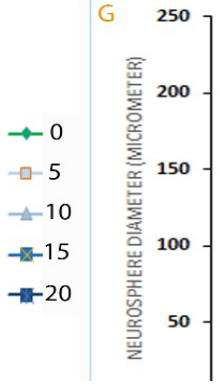

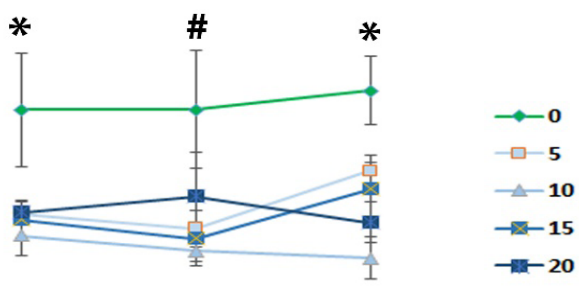

NEUR SCIENCE

The proliferation capacity of the neural stem/progenitor cells (NS/PCs) was assessed 3, 5, and 7 days after exposure to 5, 10, 15 , or $20 \mu \mathrm{g} / \mathrm{mL}$ (A-E) of methylprednisolone (MP) as the number (F) and diameter (G) of neurospheres.

$\& \mathrm{P}<0.05$ vs the 5 and 10 groups; ${ }^{*}<0.05$ vs the other groups; \# $P<0.05$ vs the other groups except for the 20 groups.

As demonstrated in the present study, previous investigations showed the anti-proliferative effect of MP. Wenhao et al. reported that the inhibitory effect of MP on the proliferation of NS/PCs is related to a decrease in the expression of hypoxia-inducible factor- $1 \alpha$ (HIF-1 $\alpha)$ and Hes1 (Wang et al., 2014). HIF-1 $\alpha$ helps the cells against apoptosis and increases cell survival (Majmundar, Wong, \& Simon, 2010). Based on the results of another study that evaluated the expression of various genes associated with neurogenesis, the anti-proliferative effect of methylprednisolone is related to the up-regulation of ferritin heavy chain 1 (Fth1) and insulin-like growth factor-binding protein (IGFBP-3) genes as well as down-regulation of endothelin receptor type B (EndrB) (Li, Wang, Tang, Huang, Wu, \& Shen, 2012). Recently, Li et al. reported that MP decreased the survival of fetal neural stem cells.

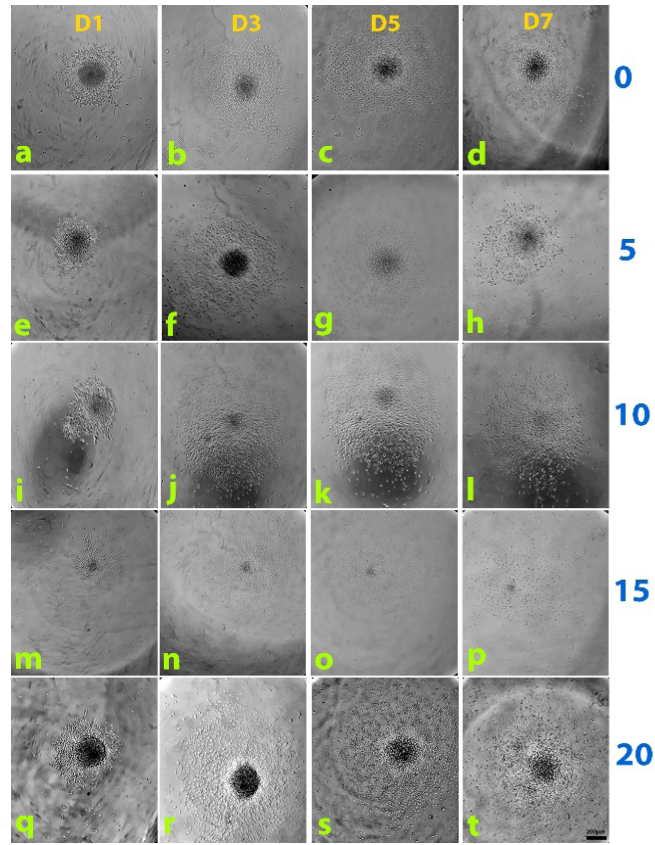

Figure 4. Cell migration assay

15
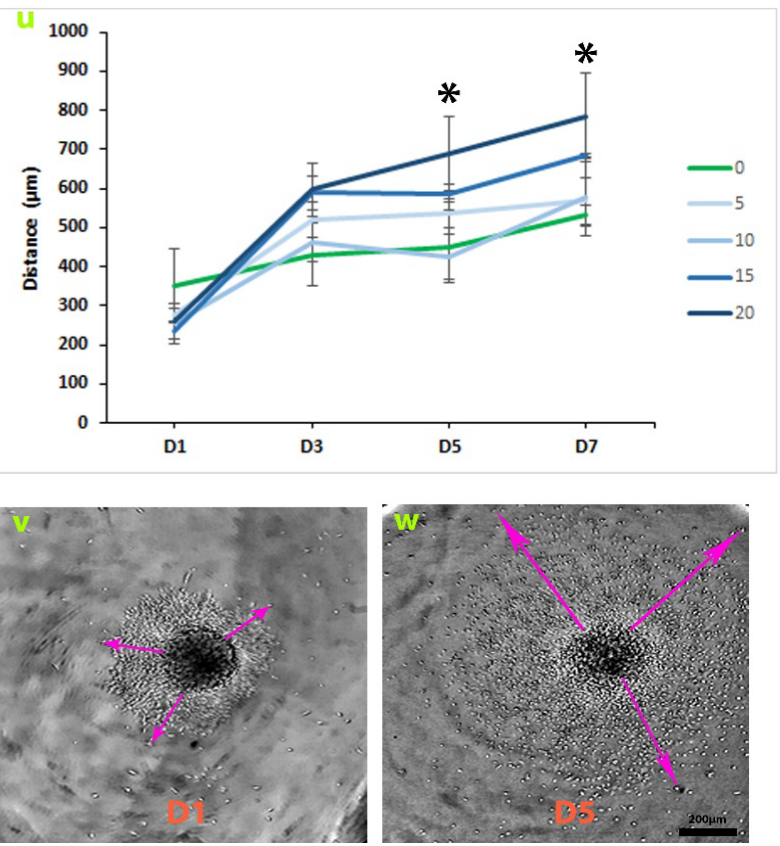

NEUR:SCIENCE

The migration of the neural stem/progenitor cells (NS/PCs) as the average distance passed by the three cells located at the farthest distance to the margin of the neurosphere was evaluated on days $1,3,5$, and 7 post-exposure to $0(\mathrm{a}-\mathrm{d}), 5(\mathrm{e}-\mathrm{h}), 10(\mathrm{i}-1), 15(\mathrm{~m}-\mathrm{p})$ or $20(q-t) \mu g / m L$ of methylprednisolone (MP). $V$ and $W$ are enlarged views of $q$ and $s$, respectively. ${ }^{*} P<0.05$ vs the 0 group. 
Using an EndrbB agonist, they explored the role of the $\mathrm{PI} 3 \mathrm{~K} / \mathrm{Akt}$ pathway and IncRNA in this adverse effect of MP (Li et al., 2020). Our investigation indicates that all studied concentrations of MP decrease the proliferation of NS/PCs. Moreover, previous studies indicate that MP inhibits the proliferation of the endogenous NS/PCs (Obermair et al., 2008). Accordingly, based on our results and previous investigations, MP inhibits the proliferation of both endogenous and exogenous NS/PCs.

The present study demonstrates the improved migration of NS/PCs following exposure to high concentrations of MP as a positive finding. The migration capacity is one of the main factors in NS/PCs transplantation (Arocena \& Collinson, 2012), reported for MP-treated NS/PCs for the first time in this study. However, further mechanistic investigations in this area are needed. However, considering the anti-survival and anti-proliferative effects of MP, its effect on migration of NS/PCs may not be beneficial combined with therapeutic approaches.

In conclusion, MP increases the migration capacity of NS/PCs only in a high concentration, but it reduces the survival and proliferation of NS/PCs. These effects of MP on NS/PCs should be considered in future combination therapies, including MP+NS/PCs for neurodegenerative diseases.

\section{Ethical Considerations}

\section{Compliance with ethical guidelines}

This study was approved by the Ethics Committee of the Shiraz University of Medical Sciences (Code: IR.SUMS.REC.1395.S34).

\section{Funding}

This research was supported by the research project (No. 8054), Funded by Shiraz University of Medical Sciences.

\section{Authors' contributions}

Conceptualization and Supervision: Hadi Aligholi; Methodology: Ahmad Soltani; Investigation, Writing - original draft, and Writing - review \& editing: All authors; Data collection: Fatemeh Shamsi, Zahra Zeraatpisheh, Mina Salmannejad; Data analysis: Zohreh Bagheri; Funding acquisition and Resources: Hadi Aligholi.

\section{Conflict of interest}

The authors declared no conflict of interest.

\section{Acknowledgments}

The authors gratefully acknowledge the support of Shiraz University of Medical Sciences (grant number 10750-74-01-94).

\section{References}

Aligholi, H., Rezayat, S. M., Azari, H., Mehr, S. E., Akbari, M \& Mousavi, S. M. M., et al. (2016). Preparing neural stem/ progenitor cells in PuraMatrix hydrogel for transplantation after brain injury in rats: A comparative methodological study. Brain Research, 1642, 197-208. [DOI:10.1016/j.brainres.2016.03.043] [PMID]

Alisky, J. M. (2008). Intrathecal corticosteroids might slow Alzheimer's disease progression. Neuropsychiatric Disease and Treatment, 4(5), 831-3. [DOI:10.2147/NDT.S3685] [PMID] [PMCID]

Almaabdi, K. H., Alshehri, R. O., Althubiti, A. A., Alsharef, Z. H., Mulla, S. N., Alshaer, D. S., et al. (2014). Intravenous methylprednisolone for intractable childhood epilepsy. Pediatric Neurology, 50(4), 334-336. [DOI:10.1016/j.pediatrneurol.2013.12.015] [PMID]

Altamentova, S., Rumajogee, P., Hong, J., Beldick, S. R., Park, S. J., \& Yee, A., et al. (2020). Methylprednisolone reduces persistent post-ischemic inflammation in a rat hypoxia-ischemia model of perinatal stroke. Translational Stroke Research, 11(5), 1117-1136. [DOI:10.1007/s12975-020-00792-2] [PMID]

Amor, S., Puentes, F., Baker, D., \& van der Valk, P. (2010) Inflammation in neurodegenerative diseases. Immunology, 129(2), 154-169. [DOI:10.1111/j.1365-2567.2009.03225.x] [PMID] [PMCID]

Arocena, M., \& Collinson, J. M. (2012). Neural stem cell migration: Role of directional cues and electric fields. In: M. A., Hayat (Eds), Stem Cells and Cancer Stem Cells (pp. 297-303). New York: Springer. [DOI:10.1007/978-94-007-4798-2_28]

Bracken, M. B., Shepard, M. J., Collins, W. F., Holford, T. R. Young, W., Baskin, D.S.,et al. (1990). A randomized, controlled trial of methylprednisolone or naloxone in the treatment of acute spinal-cord injury: Results of the second national acute spinal cord injury study. New England Journal of Medicine, 322(20), 1405-1411. [DOI:10.1056/NEJM199005173222001] [PMID]

Cummings, B. J., Uchida, N., Tamaki, S. J., Salazar, D. L., Hooshmand, M., Summers, R., et al. (2005). Human neural stem cells differentiate and promote locomotor recovery in spinal cordinjured mice. Proceedings of the National Academy of Sciences, 102(39), 14069-14074. [DOI:10.1073/pnas.0507063102] [PMID] [PMCID]

Gao, H. M., \& Hong, J. S. (2008). Why neurodegenerative diseases are progressive: Uncontrolled inflammation drives disease progression. Trends in Immunology, 29(8), 357-365. [DOI:10.1016/j.it.2008.05.002] [PMID] [PMCID]

Garbossa, D., Boido, M., Fontanella, M., Fronda, C., Ducati, A., \& Vercelli, A. (2019). therapeutic strategies for spinal cord injury 
treatment: Possible role of stem cells. Neurosurgical Review. 35(3), 293-311. [DOI:10.1007/s10143-012-0385-2] [PMID]

Gerndt, S, J., Rodriguez, J., Pawlik, J., Taheri, P., Wahl, W., \& Micheals, A., et al. (1997). Consequences of high-dose steroid therapy for acute spinal cord injury. Journal of Trauma and Acute Care Surgery, 42(2), 279-284. [DOI:10.1097/00005373199702000-00017] [PMID]

Hong Jing, Y., Ping Hou, Y., Feng Song, Y., \& Yin, J. (2012) Methylprednisolone improves the survival of new neurons following transient cerebral ischemia in rats. Acta Neurobiologiae Experimentalis., 72(3), 240-252. [PMID]

Kuppermann, B. D., Zacharias, L. C., \& Kenney, M. C. (2014) Steroid differentiation: The safety profile of various steroids on retinal cells in vitro and their implications for clinical use (an American Ophthalmological Society thesis). Transactions of the American Ophthalmological Society, 112, 116-141. [PMID] [PMCID]

Lee, J. M., Yan, P., Xiao, Q., Chen, S., Lee, K. Y., \& Hsu, C. Y., et al. (2008). Methylprednisolone protects oligodendrocytes but not neurons after spinal cord injury. Journal of Neuroscience, 28(12), 3141-3149. [DOI:10.1523/JNEUROSCI.5547-07.2008] [PMID] [PMCID]

Li, S. Y., Wang, P., Tang, Y., Huang, L., Wu, Y. F., \& Shen, H. Y. (2012). Analysis of methylprednisolone-induced inhibition on the proliferation of neural progenitor cells in vitro by gene expression profiling. Neuroscience letters, 526(2), 154-159. [DOI:10.1016/j.neulet.2012.07.047] [PMID]

Li, S., Zeng, M., Yang, L., Yang, J., Tan, J., \& Guan, H., et al (2020). EDNRB reverses methylprednisolone-mediated decrease in neural progenitor cell viability via regulating PI3K/ Akt pathway and lncRNA expression. Journal of Molecular Neuroscience, 70(3), 403-412. [DOI:10.1007/s12031-019-014274] [PMID]

Lu, P., Jones, L., Snyder, E., \& Tuszynski, M. (2003). Neural stem cells constitutively secrete neurotrophic factors and promote extensive host axonal growth after spinal cord injury. Experimental \& Neurology, 181(2), 115-129. [DOI:10.1016/S00144886(03)00037-2] [PMID]

Majmundar, A. J., Wong, W. J., \& Simon, M. C. (2010). Hypoxiainducible factors and the response to hypoxic stress. Molecular cell, 40(2), 294-309. [DOI:10.1016/j.molcel.2010.09.022] [PMID] [PMCID]

Mealey, J., Chen, T. T., \& Schanz, G. P. (1971). Effects of dexamethasone and methylprednisolone on cell cultures of human glioblastomas. Journal of Neurosurgery, 34(3), 324-334. [DOI:10.3171/jns.1971.34.3.0324] [PMID]

Obermair, F. J., Schroter, A., \& Thallmair, M. (2008). Endogenous neural progenitor cells as therapeutic target after spinal cord injury. Physiology (Bethesda), 23(5), 296-304. [DOI:10.1152/ physiol.00017.2008] [PMID]

Qian, T., Guo, X., Levi, A. D., Vanni, S., Shebert, R., \& Sipski, M. (2005). High-dose methylprednisolone may cause myopathy in acute spinal cord injury patients. Spinal Cord, 43(4), 199-203. [DOI:10.1038/sj.sc.3101681] [PMID]

Ronaghi, M., Erceg, S., Moreno-Manzano, V., \& Stojkovic, M (2010). Challenges of stem cell therapy for spinal cord injury: human embryonic stem cells, endogenous neural stem cells, or induced pluripotent stem cells? Stem Cells, 28(1), 93-99. [DOI:10.1002/stem.253] [PMID]
Russo, R. E. (2020). Functional properties of progenitor-like cells in the spinal cord: Implications for self-repair. In: G. Perale, F Rossi (Eds), Spinal Cord Injury (SCI) Repair Strategies (pp. 187202). Amsterdam: Elsevier.

Saidha, S., Mok, T., Butler, M., Fanning, N., \& Harrington, H. (2010). Multiple sclerosis exceptionally presenting as parkinsonism responds to intravenous methylprednisolone Journal of Clinical Neuroscience, 17(5), 654-655. [DOI:10.1016/j. jocn.2009.09.026] [PMID]

Sankavaram, S. R., Hakim, R., Covacu, R., Frostell, A., Neumann, S., \& Svensson, M., et al. (2019). Adult neural progenitor cells transplanted into spinal cord injury differentiate into oligodendrocytes, enhance myelination, and contribute to recovery. Stem Cell Reports, 12(5), 950-966. [DOI:10.1016/j. stemcr.2019.03.013] [PMID] [PMCID]

Sato, Y., Asoh, T., Metoki, N., \& Satoh, K. (2003). Efficacy of methylprednisolone pulse therapy on neuroleptic malignant syndrome in Parkinson's disease. Journal of Neurology, Neurosurgery \& Psychiatry, 74(5), 574-576. [DOI:10.1136/ jnnp.74.5.574] [PMID] [PMCID]

Wang, W., Wang, P., Li, S., Yang, J., Liang, X., \& Tang, Y., et al. (2014). Methylprednisolone inhibits the proliferation and affects the differentiation of rat spinal cord-derived neural progenitor cells cultured in low oxygen conditions by inhibiting HIF-1a and Hes1 in vitro. International Journal of Molecular Medicine, 34(3), 788-795. [DOI:10.3892/ijmm.2014.1835] [PMID] 
This Page Intentionally Left Blank 